\title{
Eigenvalue Decomposition Precoded Faster-Than-Nyquist Transmission of Index Modulated Symbols
}

\author{
Prakash Chaki, Takumi Ishihara, and Shinya Sugiura* \\ Institute of Industrial Science, The University of Tokyo \\ Meguro-Ku, Tokyo 153-8505, Japan \\ Email: \{prakash.chaki@ieee.org, t.ishihara@ieee.org, sugiura@iis.u-tokyo.ac.jp\}
}

\begin{abstract}
In this paper, we propose a precoded faster-thanNyquist (FTN) signaling technique for time-domain single-carrier index modulated (IM) symbol transmission. More precisely, eigenvalue decomposition precoding is adopted for the FTN transmission of data bits modulated by single-carrier time-domain IM. While the FTN scheme increases the spectral efficiency and data rate by packing more transmit symbols per block duration than those defined in the Nyquist criterion, time-domain IM works towards the same objective while maintaining symbol sparsity. We analytically derive the constrained capacity of the proposed system. Our simulation results show that the proposed scheme has better bit error ratio (BER) performance over the conventional FTN-IM scheme, particularly for the scenario of a higher packing ratio. In the proposed scheme, 2.5-dB performance gain is observed at the BER of $=10^{-4}$, employing the packing ratio of 0.7 and the roll-off factor of 0.5 in a channel-uncoded scenario.

Index Terms-Faster-than-Nyquist signaling, index modulation, eigenvalue decomposition, power allocation.
\end{abstract}

\section{INTRODUCTION}

The scarcity of communication bandwidth, as well as the demand for higher spectral efficiency, is at a record high [1], [2]. For the sake of increasing spectral efficiency, faster-thanNyquist (FTN) signaling [3] packs signal pulses tighter in time than in Nyquist signaling. Such packing of signals with high density by violating Nyquist's zero inter-symbol interference (ISI) criterion, and hence requiring advanced signal processing techniques to mitigate the deliberately introduced ISI has gained increased attention. After the original introduction of FTN signaling in [4], [5], Mazo [6] showed in his seminal work that the minimum Euclidean distance (MED) remains unaffected if the signal packing ratio $\tau$ satisfies $0.802 \leq \tau \leq 1$ for sinc pulses, hence capable of transmitting $25 \%$ faster than the rate of Nyquist signaling. In [7], the capacity of FTN signaling was derived to show that the FTN signaling is capable of the excess bandwidth. In [8], the FTN concept was extended to the frequency-domain counterpart as well as both the time-frequency domain one [9], outperforming orthogonal frequency-division multiplexing (OFDM).

Postprint accepted on 30 April 2021 for publication in IEEE International Symposium on Information Theory (ISIT), 2021. (C) 2021 IEEE. Personal use of this material is permitted. Permission from IEEE must be obtained for all other uses, in any current or future media, including reprinting/republishing this material for advertising or promotional purposes, creating new collective works, for resale or redistribution to servers or lists, or reuse of any copyrighted component of this work in other works.

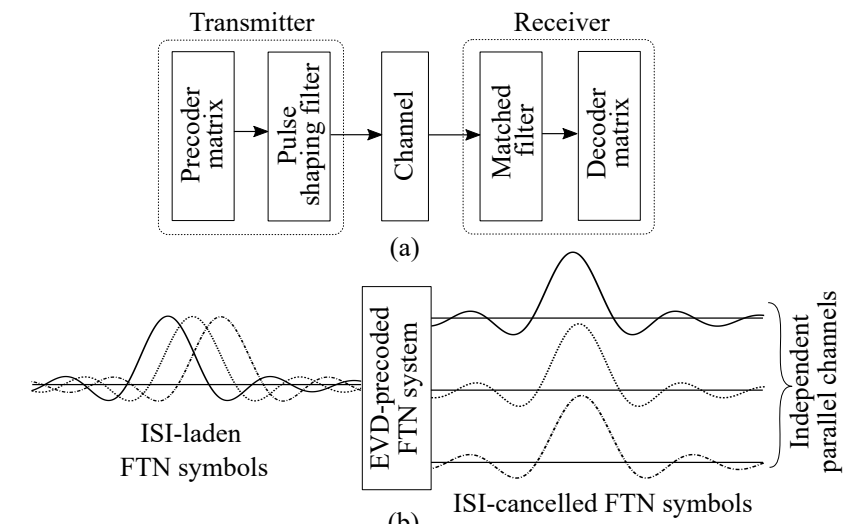

(b)

Figure 1. (a) Representative block diagram of a classic precoded-FTN system, (b) illustration of channel parallelization using EVD-precoding.

In recent years, FTN has been regarded as a promising candidate for beyond $5 \mathrm{G}$ wireless systems [10] and optical systems as well [11]. However, the improvement in spectral efficiency may be attainable at the cost of the introduction of severe ISI, which requires powerful equalization and coding techniques. Maximum-likelihood (ML) sequence estimation using the Viterbi algorithm provides the optimal decoding performance [12], although it imposes a prohibitively high complexity on the receiver. To this end, sub-optimal successive interference cancellation aided maximum a-posteriori detector was developed [7]. As a low-complexity alternative, frequency-domain equalization (FDE) was introduced in FTN [13].

Alternatively, precoding FTN signals has gained interest because of the benefits of low complexity at the receiver [14][16]. More specifically, a precoder pre-equalizes ISI specific to FTN signaling at the transmitter based on the fact that the effects of ISI are predetermined by the packing ratio and the roll-off factor $\beta$ of a shaping filter. In the linearly precoded FTN system, input symbols are multiplied by a precoding matrix in advance of pulse shaping at the transmitter, while at the received vector, the received signals are multiplied by a decoding matrix after matched filtering, as shown in Fig. 1-(a). In [16], singular-value decomposition (SVD) based precoding was discussed. The key idea behind SVD-precoded 
FTN relies on the fact that the ISI-contaminated symbol streams are decomposed into independent parallel substreams, as shown in Fig. 1-(b), similar to spatial-multiplexing multipleinput multiple-output (MIMO) channels [17, Sec. 10.2]. More recently, in [15], optimal power allocation (PA) scheme was introduced in SVD-precoded-FTN, which outperformed conventional SVD-precoded-FTN, although it operates only if the packing ratio is in the range of $1 /(1+\beta) \leq \tau \leq 1$ in a practical sense.

Index modulation [18], [19] refers to a class of modulation techniques where an activated index of communication resources (e.g., in the time, frequency, or spatial domains) is used to convey information bits, in addition to those modulated with the conventional modulation schemes, such as amplitude, frequency, and the phase of a signal. The origin of IM dates back to the classic permutation modulation [20] and parallel combinatory modulation [21]. In the 2000s, IM in the spatial domain was rediscovered as spatial modulation (SM) [22] in the context of MIMO systems in [23], [24]. SM activated only a subset of transmit antennas, and an activated antenna index is modulated as additional information. Subsequently, IM found application in multi-carrier systems as subcarrier IM (SIM) [25]. Furthermore, IM in the time domain was introduced even more recently [26], where a subset of symbols is selected for transmission, which imparts sparsity to transmit signals. It is worthy to note that time-domain IM does not require multiantenna (as in SM) or a multi-carrier (as in SIM), and hence is a low-cost technique to improve spectral efficiency. In [27], time-domain IM was exploited for FTN signaling, which is referred to as FTN-IM. To the best of our knowledge, precoded FTN-IM signaling has not been attempted despite its potential of further improving the achievable performance.

Against this backcloth, the novel contribution of this paper is that we propose a precoded FTN signaling scheme for single-carrier time-domain IM symbols. FTN and IM both complement each other in improving the spectrum efficiency since IM is based on symbol sparsity, while FTN is based on symbol density. We demonstrate that the proposed scheme using EVD-precoding aided with PA outperforms the conventional design of FTN-IM signaling. The remainder of this paper is organized as follows. Section II introduces our proposed system model, while Section III derives the constrained capacity. In Section IV, we show the simulated BER results of the proposed system and comparison with the conventional scheme. Finally, Section V concludes the paper.

Notations: We use boldface uppercase letters to denote matrices, boldface lowercase letters for vectors, and lowercase letters with a suffix to denote elements of a vector. $\mathbb{C}^{j \times k}$ and $\mathbb{R}^{j \times k}$ denote the complex and real fields of dimensions $j \times k$, respectively. Transpose and Hermitian operations are denoted by $(.)^{T}$ and $(.)^{H}$ respectively.

\section{Proposed EVD-Precoded FTN Signaling of SINGLE-CARRIER TIME-DOMAIN IM SYMBOLS}

In this section, we introduce our proposed system model. Inspired by the recent results on precoded FTN with PA [15], we design a precoded FTN signaling system with PA for timedomain IM symbols. As illustrated in Fig. 2, the proposed transmitter is constituted by the blocks of IM and APSK modulation, the blocks of PA and EVD precoding, and the block of FTN signaling, which are detailed as follows.

\section{A. IM-APSK Modulation}

Consider that random information bits are transmitted using hybrid symbols modulated based on time-domain IM and amplitude and phase-shift keying (APSK), as shown in Fig. 3. The information bits are first divided into $L$ sub-blocks, each containing $B$ bits, where each modulated sub-block corresponds to an IM-APSK symbol. In each sub-block, $B$ information bits are further divided into $B_{1}$ and $B_{2}$ bits. The first $B_{1}$ bits are modulated based on IM, where $K$ out of $M$ slots are activated, and no transmission is scheduled in the remaining $M-K$ slots, hence having the relationship of $B_{1}=\left\lfloor\log _{2}\left(\begin{array}{c}M \\ K\end{array}\right)\right\rfloor$. Then, in the activated $K$ slots, the remaining $B_{2}$ bits are modulated with the aid of $\mathcal{P}$-ary APSK, where we have $B_{2}=K \log _{2} \mathcal{P}$. Note that our IM-APSK scheme is characterized by the parameters of $(M, K, \mathcal{P})$.

Thus, we obtain an IM-APSK symbol vector of

$$
\boldsymbol{s}=\left[\left(\boldsymbol{s}^{0}\right)^{T},\left(\boldsymbol{s}^{1}\right)^{T}, \cdots,\left(\boldsymbol{s}^{L-1}\right)^{T}\right]^{T} \in \mathbb{C}^{L M},
$$

and

$$
s^{l}=\left[s_{(l)}^{0}, s_{(l)}^{1}, \ldots, s_{(l)}^{M-1}\right]^{T} \in \mathbb{C}^{M} \quad(0 \leq l<L),
$$

Here, we have the energy constraint of $\mathbb{E}\left[s^{l^{H}} s^{l}\right]=M E_{\mathrm{tx}}$, and $E_{\mathrm{tx}}$ is the average transmit energy per slot. Note that the transmit energy of activated symbols is scaled by $M / K$ to maintain the total transmit energy per block [26].

\section{B. Power Allocation and EVD Precoding}

The symbol vector $s$ is multiplied by a PA matrix $\Psi=$ $\operatorname{diag}\left\{\sqrt{\psi_{0}}, \sqrt{\psi_{1}}, \ldots, \sqrt{\psi_{L M-1}}\right\} \in \mathbb{R}^{L M \times L M}$. The powerallocated symbol vector $\Psi_{\mathrm{s}}$ is then precoded by an orthogonal matrix $\mathbf{V} \in \mathbb{R}^{L M \times L M}$, which is derived from the EVD of an FTN-induced ISI matrix [15], [16], [28], as derived in the following subsection. Thus, the precoded symbol vector is denoted as

$$
\begin{aligned}
\boldsymbol{x} & =\left[x_{0}, x_{1}, \ldots, x_{L M-1}\right]^{T} \in \mathbb{C}^{L M} \\
& =\mathbf{V} \boldsymbol{\Psi} \boldsymbol{s} .
\end{aligned}
$$

\section{FTN Signaling}

The symbols in $\boldsymbol{x}$ are band-limited using an RRC shaping filter $h(t)$ with roll-off factor $\beta$, and transmitted with an FTN symbol interval of $T=\tau T_{0}$ over the channel, where the symbol interval in an FTN signal $T$ is $\tau(<1)$ times that of the Nyquist counterpart $T_{0}$, thus transmitting more symbols in the same time interval. Throughout this paper, we maintain the transmit energy of FTN symbols $x_{i} \quad(0 \leq i<L M)$ to be the same as that of Nyquist symbols. 


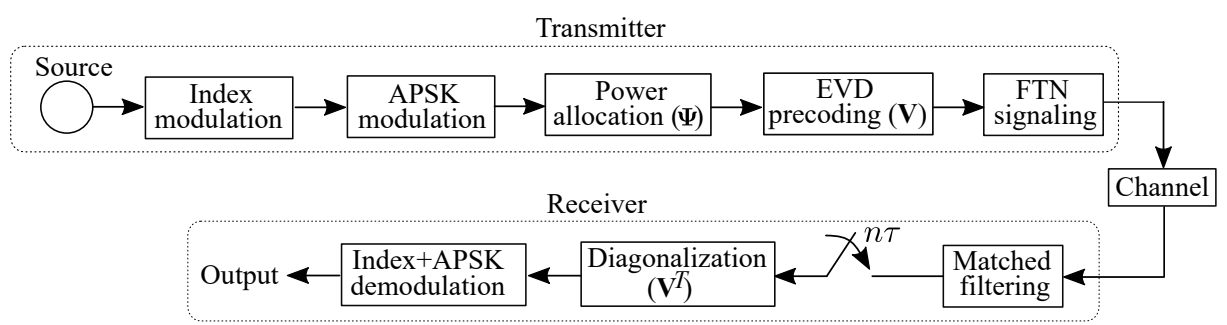

Figure 2. System model of proposed EVD-precoded FTN transmission of IM symbols.

\begin{tabular}{|c|c|c|c|c|c|c|c|c|c|c|c|c|c|c|c|}
\hline \multicolumn{4}{|c|}{ Sub-block 1} & \multicolumn{4}{|c|}{ Sub-block 2} & \multicolumn{4}{|c|}{ Sub-block 3} & \multicolumn{4}{|c|}{ Sub-block 4} \\
\hline 1 & 0 & 1 & 1 & 0 & 1 & 0 & 1 & 0 & 0 & 1 & 1 & 1 & 1 & 0 & 1 \\
\hline & & For $A$ & & & & & & & & & & & & For A & PSK \\
\hline
\end{tabular}

(a)

Active symbol transmission slots

No transmission (IM imparting sparsity to symbol transmission)
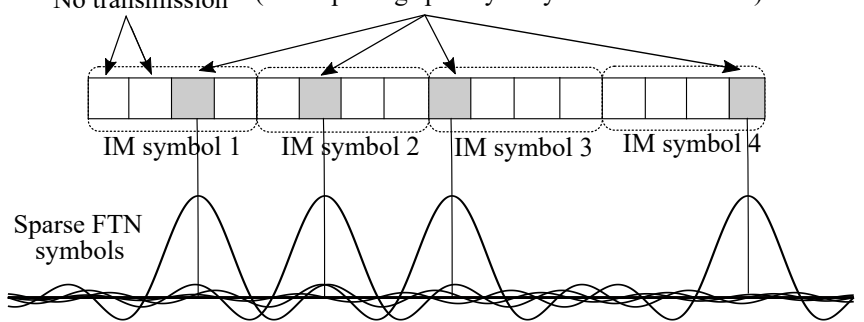

(b)

Figure 3. Exemplary illustration of the modulation scheme, where information bits of (1011010100111101) and IM parameters of $(M, K, \mathcal{P})=$ $(4,1,4)$ are considered in the proposed scheme. In each sub-block, the first two bits are modulated by IM, while the remaining two bits are modulated by the quadrature phase-shift keying (QPSK) scheme. (a) sub-block mapping and (b) generated symbols.

The continuous-time transmit signal is expressed as

$$
x(t)=\sum_{n=0}^{L M-1} x_{n} h(t-n T)
$$

For the sake of simplicity, we consider an additive white Gaussian noise (AWGN) channel. However, the proposed scheme operates in the frequency-flat fading channel without imposing any modifications. The received signal contaminated with the AWGN is represented by

$$
u(t)=\sum_{n=0}^{L M-1} x_{n} h(t-n T)+n(t),
$$

where $n(t)$ is a complex-valued white Gaussian random process with a zero mean and a spectral density of $N_{0}$. Then, $u(t)$ is processed with a matched filter $h^{*}(-t)$, and the output is given by

$$
y(t)=\sum_{n=0}^{L M-1} x_{n} g(t-n T)+\eta(t),
$$

where $g(t)=\int h(k) h^{*}(k-t) d k$ and $\eta(t)=$ $\int n(k) h^{*}(k-t) d k$. Subsequently, $y(t)$ is sampled at the FTN sampling instants $t=i T$ to obtain

$$
y(i T)=\sum_{n=0}^{L M-1} x_{n} g(i T-n T)+\eta(i T) .
$$

Moreover, (8) is rewritten in the block representation by

$$
\boldsymbol{y}=\mathbf{G} \boldsymbol{x}+\boldsymbol{\eta} \in \mathbb{C}^{L M},
$$

where $\mathbf{G} \in \mathbb{R}^{L M \times L M}$ denote the ISI matrix resulting from FTN signaling. Since $\mathbf{G}$ is a positive-definite Toeplitz matrix [15], we arrive at the EVD of $\mathbf{G}=\mathbf{V} \boldsymbol{\Lambda} \mathbf{V}^{T}$, such that $\mathbf{V} \in \mathbb{R}^{L M \times L M}$ is an orthogonal matrix and $\boldsymbol{\Lambda}=$ $\operatorname{diag}\left\{\lambda_{0}, \lambda_{1}, \ldots, \lambda_{L M-1}\right\} \in \mathbb{R}^{L M \times L M}$ is a diagonal matrix containing the eigenvalues $\lambda_{n}$ of $\mathbf{G}$. Hence, (9) is further modified to

$$
\begin{aligned}
\boldsymbol{y} & =\underbrace{\mathbf{V} \boldsymbol{\Lambda} \mathbf{V}^{T}}_{\mathbf{G}} \cdot \underbrace{\mathbf{V} \boldsymbol{\Psi} s}_{\boldsymbol{x}}+\boldsymbol{\eta} \\
& =\mathbf{V} \boldsymbol{\Lambda} \boldsymbol{\Psi} \boldsymbol{s}+\boldsymbol{\eta} .
\end{aligned}
$$

By multiplying $\boldsymbol{y}$ by $\mathbf{V}^{T}$, the received block is diagonalized as

$$
\begin{aligned}
\boldsymbol{y}^{\dagger} & =\mathbf{V}^{T} \boldsymbol{y} \in \mathbb{C}^{L M} \\
& =\boldsymbol{\Lambda} \boldsymbol{\Psi} \boldsymbol{s}+\boldsymbol{\eta}^{\dagger}
\end{aligned}
$$

where $\boldsymbol{\eta}^{\dagger}=\mathbf{V}^{T} \boldsymbol{\eta}$. Note that $\boldsymbol{\eta}$ are correlated noises, while $\boldsymbol{\eta}^{\dagger}$ are uncorrelated ones [15]. Since $\boldsymbol{\Psi}$ and $\boldsymbol{\Lambda}$ are diagonal matrices, (13) simply translates to

$$
y_{n}^{\dagger}=\sqrt{\psi_{n}} \lambda_{n} s_{n}+\eta_{n}^{\dagger} .
$$

As evident from (14), $\boldsymbol{y}^{\dagger}$ can be viewed as the output from $L M$ parallel channels (as illustrated earlier in Fig. 1-(b)), each with channel gain equal to $\sqrt{\psi_{n}} \lambda_{n}$ and additive noise $\eta_{n}^{\dagger}$, obeying the complex-valued Gaussian distribution with the zero-mean and the variance of $N_{0} \lambda_{n}$.

It may be possible to choose the values of PA coefficients $\psi_{n}$ in a manner so as to maximize mutual information. However, optimizing PA coefficients for the proposed FTN-IM signaling is non-trivial since computing the closed-form expression for the mutual information is challenging in our model. To avoid such complications, we employ the PA scheme of [15], which was shown to be optimal for non-IM FTN signaling over the AWGN channel, which is given by

$$
\psi_{n}=\frac{1}{\lambda_{n}} .
$$


Note that in [15], the optimal power coefficients of (15) are derived under the idealistic assumption that all the eigenvalues of $\mathbf{G}$ are positive even for an infinite block length $n \rightarrow \infty$. However, this may not be true unless the packing ratio is as high as $1 /(1+\beta) \leq \tau \leq 1$. Hence, in order to maintain all the positive $N$ eigenvalues, in this paper we consider only the limited packing ratio range of $1 /(1+\beta) \leq \tau \leq 1$.

Subsequently, $\boldsymbol{y}^{\dagger}$ is demodulated based on symbol-bysymbol ML detection. Let us divide $\boldsymbol{y}^{\dagger}$ as $L$ sub-blocks as follows:

$$
\boldsymbol{y}^{\dagger}=\left[\left(\boldsymbol{y}^{0}\right)^{T},\left(\boldsymbol{y}^{1}\right)^{T}, \cdots,\left(\boldsymbol{y}^{L-1}\right)^{T}\right]^{T} \in \mathbb{C}^{L M} .
$$

Then, in order to estimate information bits $\hat{\boldsymbol{b}}^{l}=\left[\hat{\boldsymbol{b}}_{1}^{l}, \hat{\boldsymbol{b}}_{2}^{l}\right]$ in the lth block, we carry out the exhaustive search of

$$
\hat{\boldsymbol{b}}^{l}=\underset{\left(\boldsymbol{b}_{1}^{l}, \boldsymbol{b}_{2}^{l}\right)}{\arg \min }\left\|\boldsymbol{y}^{\boldsymbol{l}}-\left.\boldsymbol{s}\right|_{\left(\boldsymbol{b}_{1}^{l}, \boldsymbol{b}_{2}^{l}\right)}\right\|^{2} .
$$

The transmission rate of the proposed method is given by

$$
R=\frac{1}{\tau(1+\beta)} \frac{K \log _{2} \mathcal{P}+\left\lfloor\log _{2}\left(\begin{array}{c}
M \\
K
\end{array}\right)\right\rfloor}{M} \text { bits/s/Hz. }
$$

\section{Achievable Constrained Capacity of the PROPOSED SCHEME}

Unconstrained ergodic (Shannon) capacity is given by maximizing the average mutual information (AMI) between the input and output of a continuous input continuous output memoryless channel (CCMC) under the assumption of Gaussian input symbols. However, when employing a discrete modulation technique, a more pertinent metric is the constrained AMI, which is the discrete-input continuous output memoryless channel (DCMC) capacity [29]. In this section, we formulate the DCMC capacity of our proposed scheme.

With the PA $\Psi^{*}=\Lambda^{-\frac{1}{2}}$ of (15), (13) is rewritten as

$$
\boldsymbol{y}^{\dagger}=\boldsymbol{\Lambda}^{\frac{1}{2}} \boldsymbol{s}+\boldsymbol{\eta}^{\dagger}
$$

where we have the noise covariance matrix of

$$
\begin{aligned}
\mathbb{E}\left[\boldsymbol{\eta}^{\dagger} \boldsymbol{\eta}^{\dagger^{H}}\right] & =\mathbf{V}^{T} \underbrace{\mathbb{E}\left[\boldsymbol{\eta} \boldsymbol{\eta}^{H}\right]}_{N_{0} \mathbf{G}} \mathbf{V} \\
& =N_{0} \boldsymbol{\Lambda} .
\end{aligned}
$$

Equalizing $\boldsymbol{y}^{\dagger}$ by $\boldsymbol{\Lambda}^{-\frac{1}{2}}$, we have

$$
\boldsymbol{\Lambda}^{-\frac{1}{2}} \boldsymbol{y}^{\dagger}=\boldsymbol{s}+\boldsymbol{\Lambda}^{-\frac{1}{2}} \boldsymbol{\eta}^{\dagger}
$$

The DCMC capacity of the proposed EVD-FTN-IM scheme for $M=2^{L B}$ symbols in the two-dimensional signal space is expressed as [29]

$$
\begin{aligned}
C_{\mathrm{DCMC}}^{\text {proposed }} & =\underset{p\left(\boldsymbol{s}^{0}\right) \ldots p\left(\boldsymbol{s}^{M-1}\right)}{\arg \max } \sum_{m=0}^{M-1} \int_{-\infty}^{\infty} \int_{-\infty}^{\infty} p\left(\boldsymbol{y}^{\dagger} \mid \boldsymbol{s}^{m}\right) p\left(\boldsymbol{s}^{m}\right) \\
& \times \log _{2}\left(\frac{p\left(\boldsymbol{y}^{\dagger} \mid \boldsymbol{s}^{m}\right)}{\sum_{n=0}^{M-1} p\left(\boldsymbol{y}^{\dagger} \mid \boldsymbol{s}^{n}\right) p\left(\boldsymbol{s}^{n}\right)}\right) d \boldsymbol{y}^{\dagger} \text { bits/symbol, }
\end{aligned}
$$

where the term $p\left(\boldsymbol{y}^{\dagger} \mid \boldsymbol{s}\right)$ in (23) is given by

$$
p\left(\boldsymbol{y}^{\dagger} \mid \boldsymbol{s}\right)=\frac{1}{\left(\sqrt{\pi N_{0}}\right)^{N}} \exp \left(\frac{-\left\|\boldsymbol{\Lambda}^{-\frac{1}{2}} \boldsymbol{y}^{\dagger}-\boldsymbol{s}\right\|^{2}}{N_{0}}\right) .
$$

Furthermore, for maximizing the average mutual information, the symbols are assumed to be equiprobable. Thus, the logarithm term in (23) is simplified to

$$
\begin{aligned}
& \log _{2}\left(\frac{p\left(\boldsymbol{y}^{\dagger} \mid \boldsymbol{s}^{m}\right)}{\sum_{m=0}^{M-1} p\left(\boldsymbol{y}^{\dagger} \mid \boldsymbol{s}^{n}\right) p\left(\boldsymbol{s}^{n}\right)}\right) \\
& \quad=\log _{2} M-\log _{2}\left(\sum_{n=0}^{M-1} \frac{p\left(\boldsymbol{y}^{\dagger} \mid \boldsymbol{s}^{n}\right)}{p\left(\boldsymbol{y}^{\dagger} \mid \boldsymbol{s}^{m}\right)}\right) .
\end{aligned}
$$

Moreover, based on (24), we can calculate

$$
\begin{aligned}
& \frac{p\left(\boldsymbol{y}^{\dagger} \mid \boldsymbol{s}^{n}\right)}{p\left(\boldsymbol{y}^{\dagger} \mid \boldsymbol{s}^{m}\right)} \\
& =\exp \left(\frac{\left\|\boldsymbol{\Lambda}^{-\frac{1}{2}} \boldsymbol{y}^{\dagger}-\boldsymbol{s}^{n}\right\|^{2}-\left\|\boldsymbol{\Lambda}^{-\frac{1}{2}} \boldsymbol{y}^{\dagger}-\boldsymbol{s}^{m}\right\|^{2}}{N_{0}}\right) \\
& =\exp (\underbrace{\frac{-\left\|\left(\boldsymbol{s}^{m}-\boldsymbol{s}^{n}\right)+\boldsymbol{\Lambda}^{-\frac{1}{2}} \boldsymbol{\eta}^{\dagger}\right\|^{2}+\left\|\boldsymbol{\Lambda}^{-\frac{1}{2}} \boldsymbol{\eta}^{\dagger}\right\|^{2}}{N_{0}}}_{\varphi_{m, n}}) .
\end{aligned}
$$

Finally, we arrive at the DCMC capacity of (27)-(29), which are shown on the top of the following page. Also, the DCMC capacity in the unit of bits $/ \mathrm{s} / \mathrm{Hz}$ is given by

$$
\begin{aligned}
R & =\frac{1}{\tau(1+\beta)} \\
& \times\left(\log _{2} M-\frac{1}{M} \sum_{m=0}^{M-1} \mathbb{E}\left[\log _{2} \sum_{n=0}^{M-1} \exp \left(\varphi_{m, n}\right) \mid s^{m}\right]\right),
\end{aligned}
$$

which is only valid for the packing ratio range of $1 /(1+\beta) \leq$ $\tau \leq 1$, for the above-mentioned reason.

\section{Numerical Results}

In this section, we provide our performance results to characterize the achievable performance of the proposed EVDFTN-IM scheme in the channel-uncoded scenario. Also, we considered the three benchmark schemes, i.e., the conventional FTN-IM scheme with FDE [27], SC-IM scheme with ML detection [26], and the classic Nyquist signaling scheme with ML detection, each employing the RRC filter with the roll-off factor of $\beta$ similar to the proposed scheme. The frame length was set to $L M=512$. The CP length was set to 20 for the SC-IM and FTN-IM schemes while ignoring the related SNR penalty. The IM parameters of $(M, K, \mathcal{P})=(4,1,4)$ were considered for the proposed EVD-FTN-IM, the conventional FTN-IM, and the SC-IM schemes, while BPSK was employed for the Nyquist signaling benchmark. Hence, the transmission rate of the SC-IM and the Nyquist signaling benchmarks was given by $1 /(1+\beta)$ bits $/ \mathrm{s} / \mathrm{Hz}$, while that of the proposed and conventional FTN-IM schemes was $1 / \tau(1+\beta)$. 


$$
\begin{aligned}
C_{\mathrm{DCMC}}^{\text {proposed }} & =\sum_{m=0}^{M-1} \int_{-\infty}^{\infty} \int_{-\infty}^{\infty} \frac{1}{M} p\left(\boldsymbol{y}^{\dagger} \mid \boldsymbol{s}^{m}\right) \times\left[\log _{2} M-\log _{2}\left(\sum_{n=0}^{M-1} \exp \left(\varphi_{m, n}\right)\right)\right] d \boldsymbol{y}^{\dagger} \\
& =\frac{\log _{2} M}{M} \sum_{m=0}^{M-1} \underbrace{\int_{-\infty}^{\infty} \int_{-\infty}^{\infty} p\left(\boldsymbol{y}^{\dagger} \mid \boldsymbol{s}^{m}\right) d \boldsymbol{y}^{\dagger}}_{=1}-\frac{1}{M} \sum_{m=0}^{M-1} \underbrace{\int_{-\infty}^{\infty} p\left(\boldsymbol{y}^{\dagger} \mid \boldsymbol{s}^{m}\right) \log _{2}\left(\sum_{n=0}^{M-1} \exp \left(\varphi_{m, n}\right)\right) d \boldsymbol{y}^{\dagger}}_{\mathbb{E}\left[\log _{2} \sum_{n=0}^{M-1} \exp \left(\varphi_{m, n}\right) \mid \boldsymbol{s}^{m}\right]} \\
& =\log _{2} M-\frac{1}{M} \sum_{m=0}^{M-1} \mathbb{E}\left[\log _{2} \sum_{n=0}^{M-1} \exp \left(\varphi_{m, n}\right) \mid \boldsymbol{s}^{m}\right] \text { bits/symbol. }
\end{aligned}
$$

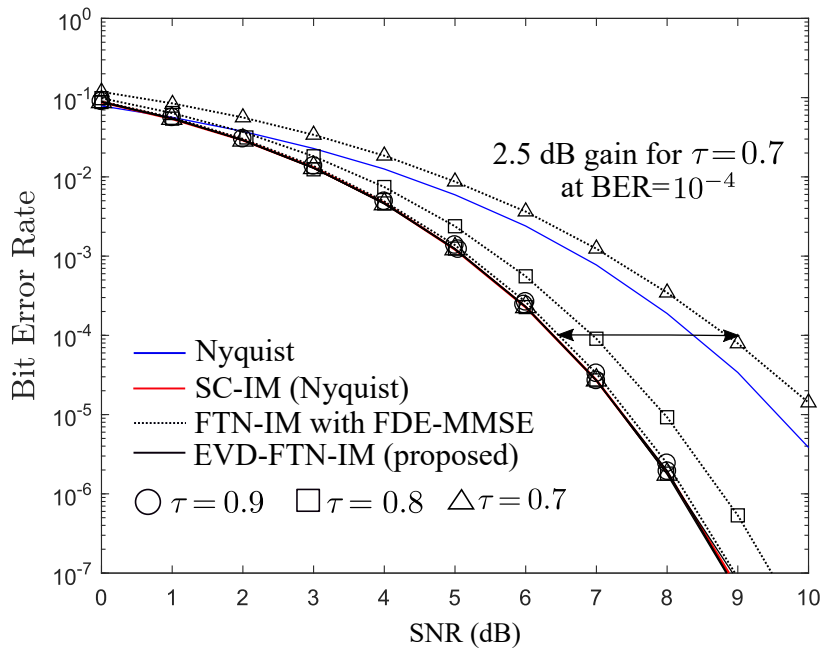

Figure 4. BER vs. SNR plot using RRC pulse of roll-off factor $\beta=0.5$.

Fig. 4 shows the BER performance for the roll-off factor of $\beta=0.5$, while the packing ratio of the proposed and conventional FTN-IM schemes was given by $\tau=0.9,0.8$, and 0.7. Observe in Fig. 4 that the proposed EVD-FTN-IM scheme exhibited better performance than the conventional FTN-IM scheme, where the performance advantage increased upon decreasing the packing ratio. More specifically, almost 2.5-dB gain was observed at the BER of $10^{-4}$ for $\tau=0.7$. Note that the BER curves of the proposed EVD-FTN-IM scheme achieved the lower bound represented by the SC-IM scheme, despite the proposed scheme's $1 / \tau(>1)$ times higher transmission rate.

Note that the proposed FTN-IM scheme requires precise calculations of the eigenvalues $\lambda_{n}$ [30]. However, significantly low eigenvalues are avoided in our scheme by choosing the packing ratio of $\tau \geq 1 /(1+\beta)$, where all the eigenvalues were accurately calculated in a double-precision environment.

Finally, Fig. 5 shows the BER performance for the roll-off factor of $\beta=0.25$, where the packing ratio was given by $\tau=$ 0.9 and 0.8. Similar to Fig. 4, it was found that the proposed scheme outperforms the conventional FTN-IM scheme by a gap of about $2.0 \mathrm{~dB}$ at the BER of $10^{-4}$ for $\tau=0.8$.

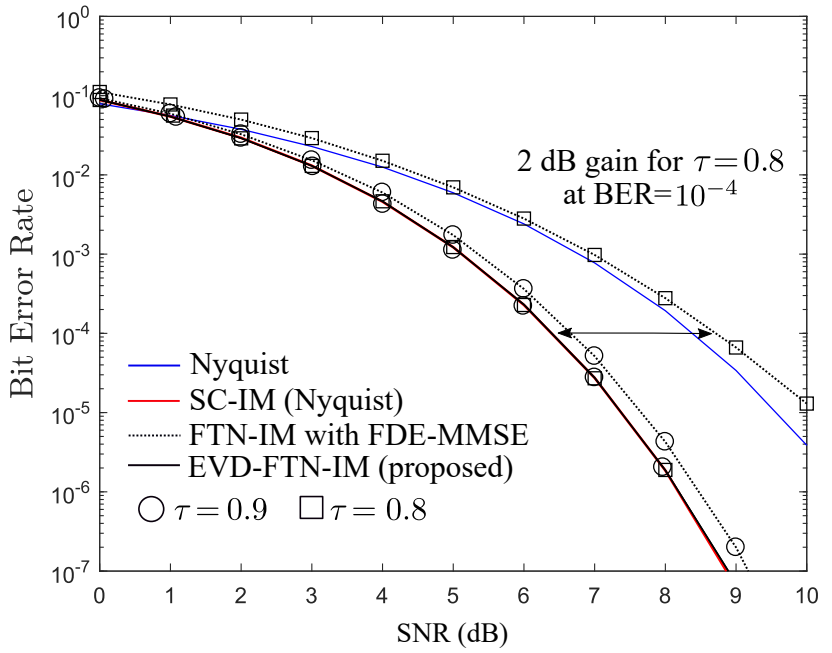

Figure 5. BER vs. SNR plot using RRC pulse of roll-off factor $\beta=0.25$.

\section{CONCLUSiON}

In this paper, we proposed the novel EVD-precoded FTNIM signaling scheme with PA, which is capable of achieving higher performance than the conventional FTN-IM scheme and Nyquist-based signaling scheme. The DCMC capacity of the proposed scheme was derived, and the numerical BER results were provided. It was demonstrated that the proposed scheme outperforms the conventional FTN-IM scheme and the classic Nyquist signaling scheme and is capable of achieving the limit of the SC-IM scheme while maintaining $1 / \tau$ times higher transmission rate.

\section{ACKNOWLEDGEMENT}

The present study was supported in part by the Japan Society for the Promotion of Science (JSPS) KAKENHI (Grant Numbers 16KK0120, 17H03259, 17K18871) and in part by JST PRESTO (Grant Number JPMJPR1933).

\section{REFERENCES}

[1] "IMT traffic estimates for the years 2020 to 2030," Report ITU-R M.2370-0 (07/2015), ITU, 2015.

[2] "Cisco annual Internet report (2018-2023)," White paper, Cisco, 2020.

[3] J. B. Anderson, F. Rusek, and V. Owall, "Faster-than-Nyquist signaling," Proceedings of the IEEE, vol. 101, no. 8, pp. 1817-1830, 2013. 
[4] D. W. Tufts, "Nyquist's problem - The joint optimization of transmitter and receiver in pulse amplitude modulation," Proceedings of the IEEE, vol. 53, no. 3, pp. 248-259, 1965.

[5] — "On "sampling, data transmission, and the Nyquist rate" and adaptive communication using randomly time-varying channels," Proceedings of the IEEE, vol. 56, no. 5, pp. 889-889, 1968.

[6] J. E. Mazo, "Faster-than-Nyquist signaling," The Bell System Technical Journal, vol. 54, no. 8, pp. 1451-1462, 1975.

[7] F. Rusek and J. B. Anderson, "Constrained capacities for faster-thanNyquist signaling," IEEE Transactions on Information Theory, vol. 55, no. 2, pp. 764-775, 2009.

[8] I. Kanaras, A. Chorti, M. R. D. Rodrigues, and I. Darwazeh, "Spectrally efficient FDM signals: Bandwidth gain at the expense of receiver complexity," in 2009 IEEE International Conference on Communications, 2009, pp. 1-6.

[9] F. Rusek and J. B. Anderson, "The two dimensional Mazo limit," in Proceedings. International Symposium on Information Theory, 2005. ISIT 2005., 2005, pp. 970-974.

[10] "5G evolution and 6G," White paper, Docomo, Jan, 2020.

[11] "White paper on technological developments of optical networks," White paper, Huawei, June, 2016.

[12] A. D. Liveris and C. N. Georghiades, "Exploiting faster-than-Nyquist signaling," IEEE Transactions on Communications, vol. 51, no. 9, pp. 1502-1511, 2003.

[13] S. Sugiura, "Frequency-domain equalization of faster-than-Nyquist signaling," IEEE Wireless Communications Letters, vol. 2, no. 5, pp. 555558, 2013.

[14] M. Maso and S. Tomasin, "Pre-equalized faster than Nyquist transmission for 5G cellular microwave backhaul," in IEEE 17th International Workshop on Signal Processing Advances in Wireless Communications (SPAWC), 2016, pp. 1-6.

[15] T. Ishihara and S. Sugiura, "SVD-precoded faster-than-Nyquist signaling with optimal and truncated power allocation," IEEE Transactions on Wireless Communications, vol. 18, no. 12, pp. 5909-5923, 2019.

[16] A. Gattami and E. Ringh, "Pre-coding in a faster-than-Nyquist transmission system," U.S. Patent 9838 230B2, Dec. 5, 2014.

[17] A. Goldsmith, Wireless communications. Cambridge University Press, 2005.

[18] S. Sugiura, T. Ishihara, and M. Nakao, "State-of-the-art design of index modulation in the space, time, and frequency domains: Benefits and fundamental limitations," IEEE Access, vol. 5, pp. 21 774-21 790, 2017.
[19] T. Mao, Q. Wang, Z. Wang, and S. Chen, "Novel index modulation techniques: A survey," IEEE Communications Surveys Tutorials, vol. 21, no. 1, pp. 315-348, 2019.

[20] D. Slepian, "Permutation modulation," Proceedings of the IEEE, vol. 53, no. 3, pp. 228-236, 1965.

[21] S. Sasaki, Jinkang Zhu, and G. Marubayashi, "Performance of parallel combinatory spread spectrum multiple access communication systems," in IEEE International Symposium on Personal, Indoor and Mobile Radio Communications., 1991, pp. 204-208.

[22] M. Di Renzo, H. Haas, A. Ghrayeb, S. Sugiura, and L. Hanzo, "Spatial modulation for generalized MIMO: Challenges, opportunities, and implementation," Proceedings of the IEEE, vol. 102, no. 1, pp. 56-103, 2014.

[23] Y. A. Chau and Shi-Hong Yu, "Space modulation on wireless fading channels," in IEEE 54th Vehicular Technology Conference, vol. 3, 2001, pp. 1668-1671.

[24] R. Y. Mesleh, H. Haas, S. Sinanovic, C. W. Ahn, and S. Yun, "Spatial modulation," IEEE Transactions on Vehicular Technology, vol. 57, no. 4 pp. 2228-2241, 2008.

[25] R. Abu-alhiga and H. Haas, "Subcarrier-index modulation OFDM," in IEEE 20th International Symposium on Personal, Indoor and Mobile Radio Communications, 2009, pp. 177-181.

[26] M. Nakao, T. Ishihara, and S. Sugiura, "Single-carrier frequency-domain equalization with index modulation," IEEE Communications Letters, vol. 21, no. 2, pp. 298-301, 2017.

[27] T. Ishihara and S. Sugiura, "Faster-than-Nyquist signaling with index modulation," IEEE Wireless Communications Letters, vol. 6, no. 5, pp. 630-633, 2017.

[28] B. Qian, X. Wang, J. Wen, S. Zhang, and C. Chen, "Novel intersymbol interference cancellation scheme to enable parallel computational and high-performance faster-than-Nyquist signaling," IEEE Access, vol. 5, pp. 24 758-24 765, 2017.

[29] S. X. Ng and L. Hanzo, "On the MIMO channel capacity of multidimensional signal sets," IEEE Transactions on Vehicular Technology, vol. 55, no. 2, pp. 528-536, 2006.

[30] K. Masaki, T. Ishihara, and S. Sugiura, "Tradeoff between calculation precision and information rate in eigendecomposition-based faster-thanNyquist signaling," IEEE Access, vol. 8, pp. 223 461-223 471, 2020. 